www.nature.com/ejhg

\title{
Both common and unique susceptibility genes in different rat strains with pristane-induced arthritis
}

\author{
Shemin Lu $^{\star, 1,3}$, Niklas Nordquist ${ }^{2,3}$, Jens Holmberg ${ }^{1}$, Peter Olofsson ${ }^{1}$, Ulf Pettersson ${ }^{2}$ and \\ Rikard Holmdahl ${ }^{1}$
}

${ }^{1}$ Section for Medical Inflammation Research, Biomedical Center, Lund University, Lund, Sweden; ${ }^{2}$ Section for Medical Genetics, Department of Genetics and Pathology, Uppsala University, Uppsala, Sweden

Pristane-induced arthritis (PIA) in rats is an animal model for rheumatoid arthritis (RA). We have previously identified seven quantitative trait loci (QTLs), which regulate arthritis development using a cross between the susceptible DA strain and the resistant E3 strain of rats (Pia2 - 8). In the present study the inbred rat strain LEW.1F was used as the susceptible strain in a cross with the E3 strain. The results confirmed the locus Pia4 on chromosome 12, which previously was shown to be associated with PIA, and also with experimental allergic encephalomyelitis, in crosses between the rat strains E3 and DA. On chromosome 1, linked to the albino locus, we identified a novel QTL, Pia9 in the LEW.F1 cross. This locus was associated with arthritis severity in the early phase of disease. A locus on chromosome 16, denoted Pia11, was also associated with arthritis severity in the early phase of the disease. A suggestive locus was detected on chromosome 14, which was associated with arthritis severity at the time when PIA progresses into a chronic phase. Using a congenic LEW.1F strain, which carries E3 alleles at the Pia9 locus, we confirmed that the E3 allele significantly suppresses arthritis severity during the early phase of the disease. The results revealed synergistic effects between different susceptibility loci using ANOVA analysis. These interactions were influenced by gender. Rats with Pia9 alleles from LEW.1F and Pia11 alleles from E3, were shown to suffer from much more severe arthritis in the early stage of the disease. On the other hand, the Pia9 and the suggestive locus on chromosome 14 affected only males during the chronic phase of the disease. These findings provide clues to how genetic factors by themselves, and in interaction with each other, regulate the development of a disease, which displays many similarities to RA.

European Journal of Human Genetics (2002) 10, 475 -483. doi:10.1038/sj.ejhg.520832

Keywords: pristane-induced arthritis; rheumatoid arthritis; QTL; rats; linkage; autoimmune disease; genetics

\section{Introduction}

Rheumatoid arthritis (RA) is considered to be an autoimmune disease affecting about $1 \%$ of the population worldwide. ${ }^{1}$ The etiology of this complex disorder is unknown, and the genetic contribution is obscured by strong environmental factors. ${ }^{2}$ The most intensively studied genetic factor associated with RA is the major histocompat-

*Correspondence: S Lu, Section for Medical Inflammation Research, Sölvegatan 19, 111 BMC, Lund University, S-22184 Lund, Sweden. Tel: +46-46-2223339; Fax: +46-46-2223110;

E-mail: Shemin.Lu@inflam.lu.se

${ }^{3}$ These authors contributed equally to this study.

Received 23 November 2001; revised 25 April 2002; accepted 2 May 2002 ibility complex (MHC, or HLA in humans). ${ }^{3-7}$ The MHC accounts for $30-60 \%$ of the variability, based on $\lambda_{\mathrm{MHC}}$ estimates in various populations. ${ }^{8-11}$ RA is considered to be an autoimmune. The genetic regulation of autoimmune diseases is poorly understood and at least three models have been proposed, a multiplicative model, $^{12}$ a polygenic threshold liability model ${ }^{13}$ and a heterogeneity model. ${ }^{14}$

Recently, two whole-genome scans, involving a wide range of RA families in Europe and US, identified linkage to several chromosomal regions, including the MHC class II region. However, only the linkage to the MHC-region reached significance at the genome wide level. ${ }^{15,16}$ The 
completion of the human genome sequence gives an impetus to identify genes involved in complex diseases like RA. RA is, however, a heterogeneous disease with low penetrance, influenced by different environmental factors. Moreover, the prevalence in different ethnic populations varies. This makes it exceedingly difficult to clone the predisposing genes based on genetic studies of RA patients. An alternative is to use animal models, which allow the environmental factors as well as the genetic background to be controlled.

Several animal models for RA are well established in mice and rats, including mycobacteria-induced arthritis (MIA), collagen-induced arthritis (CIA), oil-induced arthritis (OIA), pristane-induced arthritis (PIA), and proteoglycaninduced arthritis (PgIA). ${ }^{17}$ These models mimic the human disease in many respects. Genetic studies of the models have demonstrated that MHC II genes play a crucial role in the development of the disease, as is the case for RA. An important advantage is that the animal models can be used to identify predisposing genes outside of the MHC locus. A large number of susceptibility loci have been identified which regulate arthritis development based on studies of CIA and PgIA in mice, and CIA, PIA, MIA and OIA in rats. ${ }^{13,18-22}$ These findings infer that the genetic regulation of arthritis is much more complicated than previously anticipated. There are, for instance, at least nine loci that control time of onset, severity, and chronicity of PIA in rats. ${ }^{18,23}$ Another important finding was that some loci seem to be shared among animal models for different autoimmune disorders, and also shared among different species, like mice, rats, and even humans. ${ }^{18,24-28}$ Finally, the effect of the QTLs depends on strain, gender, and different induction protocols and other environmental factors.

To limit the complexity we have selected the PIA model, to study the segregation of genetic factors in crosses between different susceptible and resistant animal strains. PIA is induced by pristane, a non-immunogenic adjuvant, and resembles RA in many aspects. ${ }^{29}$ Susceptible rat strains were injected with pristane intradermally, and the animals subsequently developed arthritis about 2 weeks after injection. The pathological changes included inflammatory cell infiltration, pannus formation, erosion and formation of bone and cartilage similar to what is seen in the joints of RA patients. It is known that $\alpha \beta$ T cells play a crucial role in the development of the disease, based on studies showing that antibody ablation can block the disease. Another important finding is that PIA, like RA, is associated with MHC. ${ }^{29}$ It is noteworthy that the association to MHC was only observed in the chronic phase of the disease. MHC is, however, not the only locus controlling the disease, shown by the fact that the arthritis incidence varies among strains with identical MHC genes on different genetic backgrounds. Two crosses involving DA and E3 and DA and the recombinant inbred strain DXEC, respectively, ${ }^{18,23}$ revealed seven QTLs. It is intriguing that among these loci, some control the arthritis onset (Pia2 and Pia3), some regulate arthritis severity (Pia4, Pia7 and Pia8), whereas others regulate chronicity (Pia5 and Pia6). Although the crosses $\mathrm{DA} \times \mathrm{DXEC}$ and $\mathrm{E} 3 \times \mathrm{DA}$ involve animals with very similar phenotypes, Pia7 and Pia8 were only revealed in the former cross, indicating complex interactions between the predisposing genes. Another locus on chromosome 10, denoted Pia10, was identified in a strain congenic for the Cia4 locus (Ron Wilder, personal communication).

In this study, we used a cross between the susceptible strain LEW.1F and the resistant strain E3 to produce 181 F2 progeny. Arthritis was induced with pristane and four QTLs were found to regulate susceptibility to arthritis. Three loci, which have not been identified previously, were identified on chromosomes 1, 14 and 16, denoted Pia9, Piax and Pia11, respectively. The fourth locus, Pia4, on rat chromosome 12 has already been shown to be an important susceptibility locus for PIA in the $\mathrm{E} 3 \times \mathrm{DA}$ cross, and displayed the same pattern of inheritance as seen previously.

\section{Materials and methods \\ Rats}

E3 and LEW.1F rats originating from the Zentralinstitut für versuchstierzucht, Hannover, Germany, were kept and bred in a climate-controlled environment with $12 \mathrm{~h}$ light/dark cycles in our animal department under pathogen-free conditions. The rats were housed in polystyrene cages, each with two or three rats, containing wood shavings and fed standard rodent chow and water ad libitum. The E3 and LEW.1F rats were intercrossed to obtain $(\mathrm{E} 3 \times \mathrm{LEW} .1 \mathrm{~F}) \mathrm{F} 1$ and F2 progeny. All experiments were performed with rats aged from 8 to 12 weeks. The rats were checked for the common pathogens including Sendai virus, Hantavirus, coronnavirus, reovirus, cytomegalovirus and mycoplasma pulmonis.

\section{Induction and evaluation of arthritis}

Pristane (2, 6, 10, 14-tetramythylpentadecane) (Aldrich, Milwaukee,WI, USA), $150 \mu \mathrm{l}$ per rat, was injected intradermally at the base of the tail under ether anaesthesia. Arthritis development was monitored using a macroscopic scoring system for each of the four limbs ranging from 0 to 4 as follows: $1=$ swelling and/or redness in one joint; $2=$ two joints involved; $3=$ more than two joints involved; and $4=$ severe arthritis in the entire paw. The maximum total score is thus 16 for each rat by adding up the scores from four paws. The rats were observed 1-3 times a week during the experimental period.

\section{Genotyping}

DNA was prepared according to a standard protocol. Primer sequences for rat microsatellite markers defined as $\mathrm{D} \times$ Mity, $\mathrm{D} \times$ Raty and $\mathrm{D} \times$ Goty were from Research Genetics (Huntsville, $\mathrm{AL}, \mathrm{USA}$ ), and for markers defined as $\mathrm{D} \times$ Woxy from the Wellcome Institute for Human Genetics (Oxford, UK). 
Fluorescent-labelled primers were synthesised by Interactiva Biotechnologie (Ulm, Germany). All the markers were assayed by PCR on ABI 877 Integrated thermal cycler (Perkin-Elmer) according to standard protocol; and PCR products were separated on an ABI 377 DNA Sequencer (Perkin-Elmer) and data analysed with software package GeneScan ${ }^{\circledR} 3.1$ and Genotyper ${ }^{\circledR} 2.1$ (Perkin-Elmer). For making the congenic strain, genotyping was performed using MegaBACE 1000 DNA Analysis System (Molecular Dynamics, USA) and data were analysed with software Genetic Profiler Version 1.1 (Molecular Dynamics, USA)

\section{Arthritis induction in the Pia9 congenic strain}

To make the strain congenic for Pia9, the conventional strategy was used to introgress the resistant region from E3 rats onto the susceptible background of LEW.1F. During the first four generations, we selected black and white rats to backcross with LEW.1F, because the c-locus was found to co-segregate with the disease. Then a panel of microsatellite markers around the D1Rat50 was used to further select rats for backcrossing. The experiment was performed on N8 rats that contained an E3 fragment between c-locus and D1Mgh21 $(<20 \mathrm{cM})$. The rats were injected with $100 \mu \mathrm{l}$ pristane intradermally in the base of the tail. Arthritis development was monitored 1-3 times until day 49 after pristane injection with the new macroscopic scoring system for the four limbs ranging from 0 to 15 points for each paw (1 point for each swollen or red toe, 1 point for mid-foot digit and knuckle, 5 points for a swollen ankle or wrist joint). In this way the scores of the four paws yields a maximum total score of 60 for each rat.

\section{Statistical analysis}

Linkage analysis was performed with the Mapmaker computer package. $^{30}$ A logarithm of odds (LOD) 3.9 was considered to be significant and above 2.8 as suggestive linkage, which is based on the guidelines suggested by Lander and Kruglyak. ${ }^{31}$ And we also used Map Manager QTXb13 software to perform permutation tests (http:// mapmgr.roswellpark.org/mmQTX.html). In order to compare incidences between males and females, the Chi- square test was performed. To compare the arthritis severity, indicated as clinical scores at the different time point after pristane injection, non-parametric analyses, Mann-Whitney test for two groups, and Kruskal-Wallis test for three groups or more, were used. Two-way ANOVA was used to test for interactions between two loci on the arthritis development. In all the analyses we used $\alpha=0.05$. The analyses were performed with the statistic software - StatView version 5.0.1 (SAS Institute, USA).

\section{Results}

Arthritis development in parental, F1, and F2 rats

The susceptibility to PIA of parental strains and the progeny from different crosses is presented in Table 1 and Figure 1. The LEW.1F rats were susceptible to PIA with an incidence of $100 \%$ in females, whereas E3 rats were completely resistant to PIA. The F1 rats were susceptible to PIA, but with a delayed onset, reduced severity and incidence. In the F1 population, only females developed disease, confirming the female preponderance in the LEW.1F strain. The F2 population exhibited a large variation in disease development after pristane injection. The severity of the disease was only slightly greater in females than in males.

\section{Identification of arthritis QTLs}

Four QTLs were identified in this cross and the results are shown in Table 2 and Figure 2. The detailed linkage map about microsatellite markers can be found in our homepage, http://net.inflam.lu.se. Pia4 on chromosome 12, discovered previously in an $(\mathrm{E} 3 \times \mathrm{DA}) \mathrm{F} 2$ intercross, was identified also in this cross, confirming the presence of a protective allele in E3. ${ }^{18}$ In LEW.1F, as in DA, this locus regulated the clinical severity in both males and females (Figure 3C and D). Novel QTLs showing significant linkage with arthritis severity were found on chromosomes 1 (Pia9), and 16 (Pia11). In addition a locus on chromosome 4 (Piax) showed suggestive linkage (Figure 2). The predisposing allele at the Pia9 locus originated from the LEW.1F strain, whereas at the Pia11 the predisposing allele was derived from the resistant E3 strain (Figure 3). The locus on chromosome 14 (Piax), displayed overdominance, ie the

Table 1 Arthritis development in parental strains, F1 and F2 rats

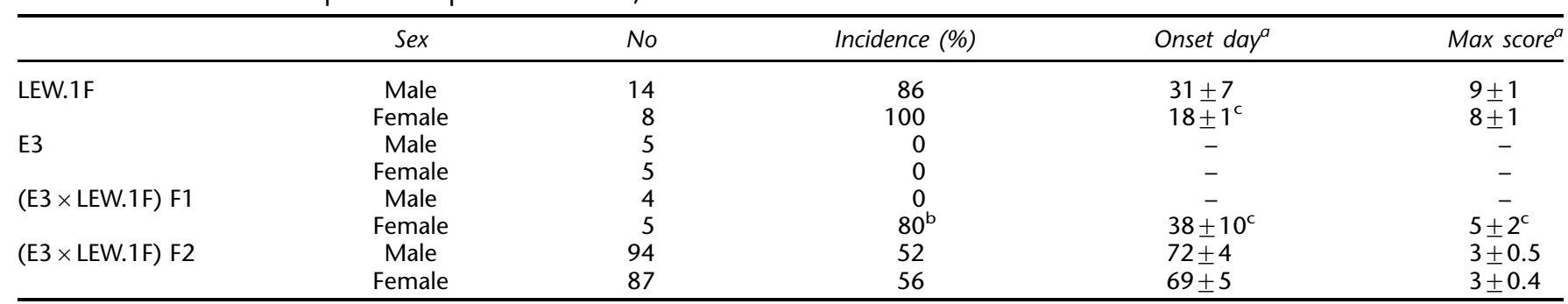

Including all rats and expressing mean \pm SEM; ${ }^{\mathrm{b}} P<0.05$ between male and female rats using Fisher's Exact Chi Square Test; ${ }^{\mathrm{c}} P<0.05$ between male and female rats using nonparametric Mann-Whitney Test. 

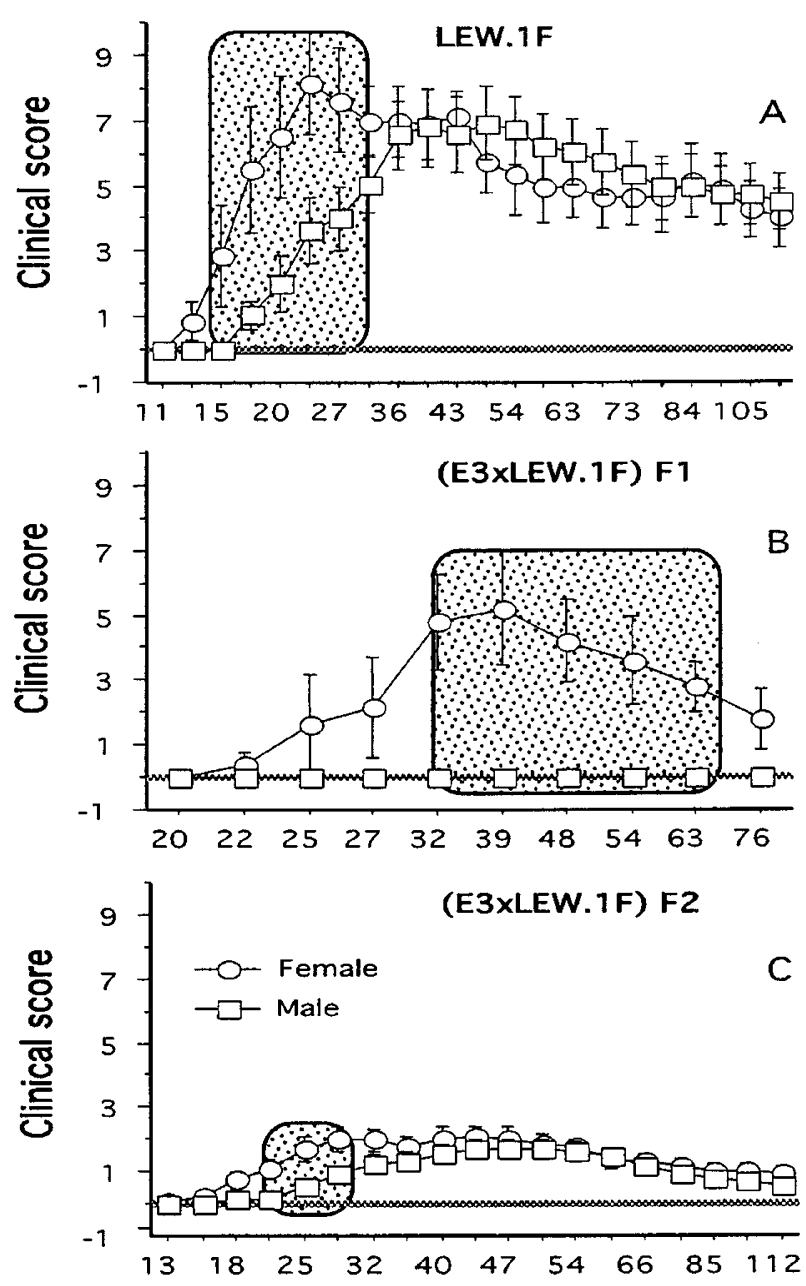

\section{Days after pristane injection}

Figure 1 Clinical scores (mean \pm SEM) and in the LEW.1F (A) $($ E3 $\times$ LEW.1F)F1 (B) and F2 rats (C) after pristane injection (150 $\mu \mathrm{l} / \mathrm{rat})$. Arthritis was monitored twice or thrice a week using a macroscopic scoring system for each of the four limbs ranging from 0 to 4 . Shaded area represents a significant difference between females and males (Mann-Whitney test, $P<0.05)$. heterozygotes had a more severe phenotype than either of the homozygotes (Figure 3). Gender differences were observed for the penetrance of the different QTLs; Pia9 and Piax had a more pronounced effect in females and Pia11 in males (Figure 3). It is showed that Pia9 regulates the severity in early stage of the disease while others control disease severity in fully flaring phase (Figure 4). The loci should obviously be confirmed in congenic strains, which is a time consuming process. So far we have tested the Pia9 locus after backcrossing of the protective E3 allele into LEW.1F for eight generations. The arthritis phenotype could be produced in the congenic strain, manifesting significantly less severe in the congenic animals (24 rats in total); but the data could not exclude the possibility that the Pia9 locus in fact contains more than one gene associated with arthritis severity (Figure 5).

\section{Interactions between the observed QTLs}

We used two-way ANOVA to study interactions between the loci, choosing the most significant markers representing each locus after single marker analyses (Kruskal-Wallis test). The combined effects of pairs of loci on the clinical scores during different phases of the disease were measured. We found that the combination of Pia9 and Pia11 showed a synergistic effect. This effect was observed in both females and males during the acute phase of the disease (Figure $6 \mathrm{~A}$ and $\mathrm{B})$. Animals homozygous for the LEW.1F allele at the Pia9 locus, and homozygous for the E3 allele at Pia11 showed a more severe disease at day 18 after pristane injection than individuals homozygous at only one of these loci (Figure 6C). The combination between Pia9 and Piax, showed a more complex pattern. Firstly, the combined effect could only be detected in male rats (Figure 7A and B). Secondly, male rats heterozygous at Pia9 or Piax, suffered from much more severe arthritis (Figure 7C) later in the disease course in contrast to the females (Figure 7D).

\section{Discussion}

PIA is controlled by several genes as previously shown by segregation analysis of the $(\mathrm{E} 3 \times \mathrm{DA})$ and $(\mathrm{DA} \times \mathrm{DXEC})$

Table 2 Linkage of arthritic traits in chromosome regions with inherit modes in female and male rats from (E3 $\times$ LEW.1F) intercross

\begin{tabular}{|c|c|c|c|c|c|c|c|c|c|}
\hline QTLS & Chromosome & Affected Phenotype ${ }^{b}$ & Marker $^{c}$ & all & $\begin{array}{l}\text { Lod scor } \\
\text { females }\end{array}$ & e males & Inheritance pattern $^{d}$ & $\begin{array}{l}\text { Variance explained } \\
\text { (\%) }\end{array}$ & Cosegerated phenotypes \\
\hline Pia9 & 1 & Score day28 & D1Mit2 & 5.3 & 3.7 & 2.2 & LEW recessive & 13 & $\begin{array}{l}\text { Max score }(4.2) \\
\text { Total score }(4.5)\end{array}$ \\
\hline $\begin{array}{l}\text { Piax }^{a} \\
\text { Pia11 }\end{array}$ & $\begin{array}{l}14 \\
16\end{array}$ & $\begin{array}{l}\text { Score day47 } \\
\text { Score day35 }\end{array}$ & $\begin{array}{l}\text { D14Rat64 } \\
\text { D16Rat53 }\end{array}$ & $\begin{array}{l}3.3 \\
4.4\end{array}$ & $\begin{array}{l}2.0 \\
2.8\end{array}$ & $\begin{array}{r}<2.0 \\
3.4\end{array}$ & $\begin{array}{l}\mathrm{h}>\mathrm{b}>\mathrm{a} \\
\mathrm{E3} \text { recessive }\end{array}$ & $\begin{array}{r}8 \\
19\end{array}$ & $\begin{array}{l}- \\
\text { Max score }(3.1) \\
\text { Total score }(3.0)\end{array}$ \\
\hline
\end{tabular}

abeing called Piax since Lod score is less than 3.9; ${ }^{b}$ Lod score of clinical score at different days after prestane injection is shown in Figure 4;

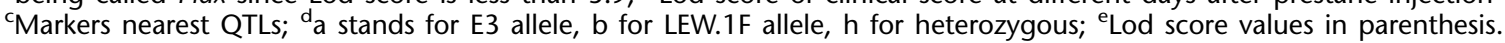



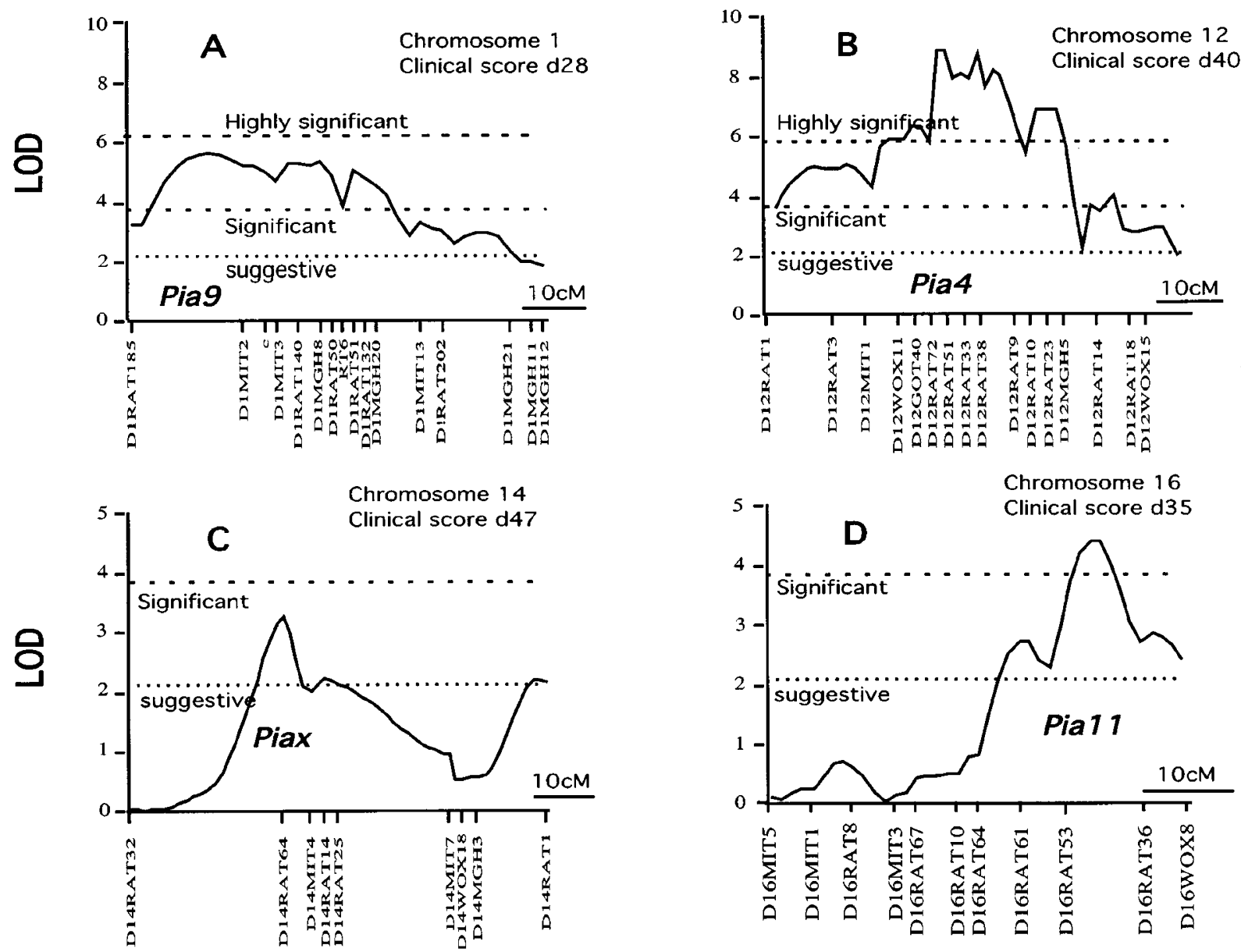

Figure 2 LOD score plots for chromosomes indicating identified OTLs. (A-D) represent LOD scores for Chromosome 1, 12, 14 and 16, respectively. The thresholds for suggestive, significant and highly significant linkages were determined after permutation test by using Map Manager QTXb13 software and indicated by dotted lines.

intercrosses. ${ }^{18,23}$ In this study, we used LEW.1F as the susceptible counterpart in a cross with the resistant strain E3, in order to study whether the same susceptibility loci were unraveled when different susceptible strains were used in the crosses. The Pia4 locus which is on rat chromosome 12, was also identified in this cross and influenced the severity of the disease. The predisposing allele originated from the susceptible strain as was the case in the $\mathrm{DA} \times \mathrm{E} 3$ cross. Three novel QTLs were identified in the LEW.F1 cross which all influence arthritis development, were denoted Pia9, Piax and Pia11, and located on chromosomes 1, 14, and 16, respectively. The QTLs detected in the current study were all influenced by gender and were associated with arthritis severity. They interacted with each other and colocalise with QTLs of other autoimmune diseases.

Females are more susceptible to PIA, as also is the case for RA. Female rats of the susceptible parental strain, LEW.1F, have much higher clinical scores both during the acute and the chronic phases, but the incidence was almost the same in the males. A female preponderance is in fact seen in both LEW.F1 and DA in other arthritis models such as avridine-induced arthritis and the type II collagen induced arthritis. ${ }^{32}$ However, this rule has exceptions since male rats are more susceptible to collagen type XI induced arthritis than female rats. ${ }^{33}$ The observed gender difference appears thus to be disease specific and does not differ between the DA and LEW strains. The mechanisms behind sex association are likely to be complex, involving both sex chromosomes and sex hormones. Clearly, sex-specific effects on autosomal QTLs are commonly observed. ${ }^{34-37}$

Previous studies have shown that the onset, severity, and chronicity of PIA are controlled by different sets of genes. In this study, we found that all loci were associated with severity although they tended to influence the phenotype at different time points. The onset of PIA in the LEW.1F is about 21 days after pristane injection, one week later than in the DA rats. Linkage to Pia9 on chromosome 1 was detected on day 18 and reached the maximum LOD 


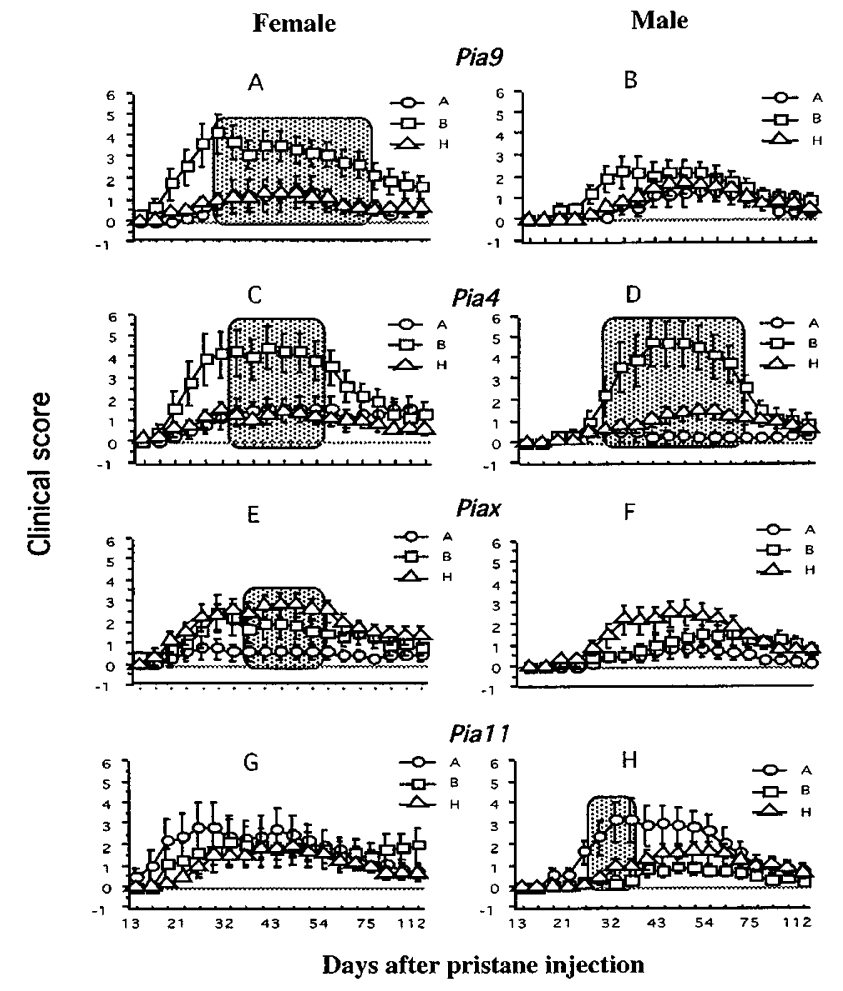

Figure 3 Disease severity with different genotypes in females and males ( $\overline{\mathbf{x}} \pm$ SEM). (A) stands for E3 homozygous, (B) for LEW.1F homozygous and $(\mathrm{H})$ for heterozygous for linked markers (same as Figures 6 and 7). Shaded areas indicated the a significant difference among different genotypes (KruskalWallis test, $P<0.05)$.

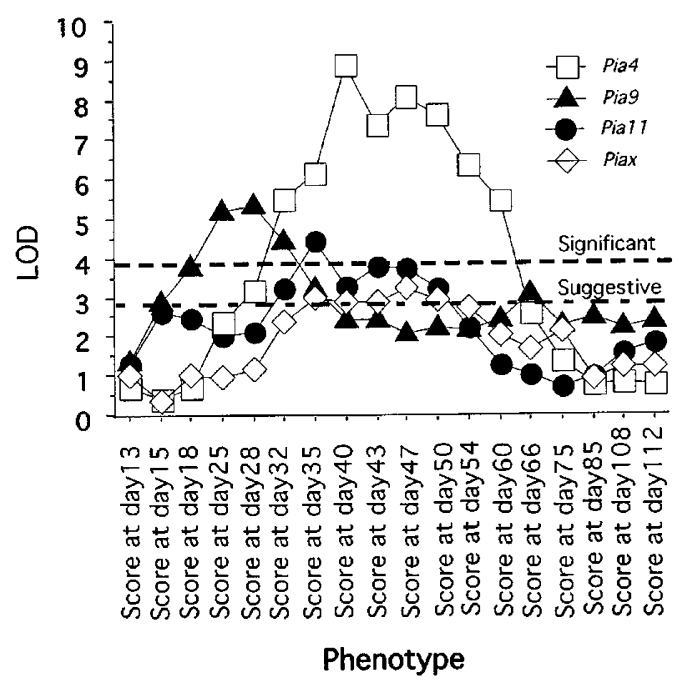

Figure 4 Lod score of 4 Pia OTLs with clinical score at different days after pristane injection. Suggestive and significant thresholds were 2.8 and 3.9 respectively suggested by Lander and Kruglyak. ${ }^{31}$

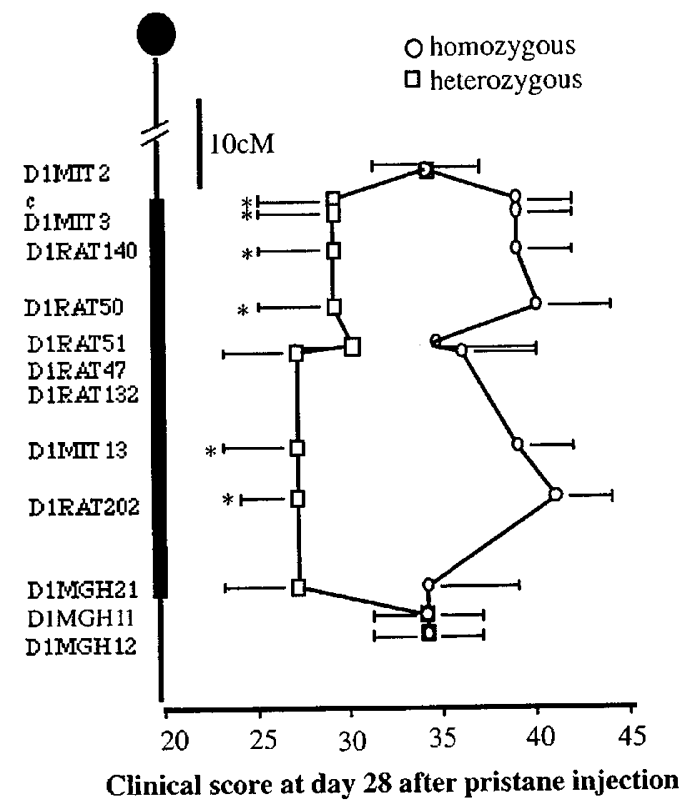

Figure 5 Confirmation of Pia9 in congenic rats $(n=24)$. The E3 fragment of Pia9 has been backcrossed on the LEW.1F background and the Pia9 heterozygous rats are compared with wild type LEW.1F rats for PIA development at day 28 after pristane injection at different markers along the congenec fragment. ${ }^{*} P<0.05$ analysed with Mann -Whitney test.

on day 28. Pia11 is also manifested early after pristane injection. Thus two loci seem to control the severity of the disease in the early phase. The suggestive locus, Piax on chromosome 14, seemed to act later in the disease. This was striking when it was analysed together with Pia9. Piax maps close to a previously identified locus associated with chronic arthritis, Pia6, but shows a different inheritance pattern. The observation that different genes are involved in the pathogenesis of PIA suggests that several different pathways are involved in disease development. It is also noteworthy that interactions between loci seem to be important for the arthritis development.

It is well known that the complex disease traits are polygenic. In this study, we utilised two-way ANOVA to analyse the pair-wise interactions between loci on the clinical scores during the course of the disease. Two loci combinations played crucial roles at the onset and during the chronic phase of the disease, respectively. One is the combination of Pia9 and Pia11. This effect is seen in both female and male rats during the acute phase of the disease (Figure 6). Another interaction between Pia9 and Piax regulated severity later in the disease course. Interestingly, when Pia9 was combined with different QTLs it manifested different effects not only in different sexes but also during various phases of the disease. It will thus be necessary to construct double congenic animals to study the interactions, as has been done successfully in the SLE mouse 

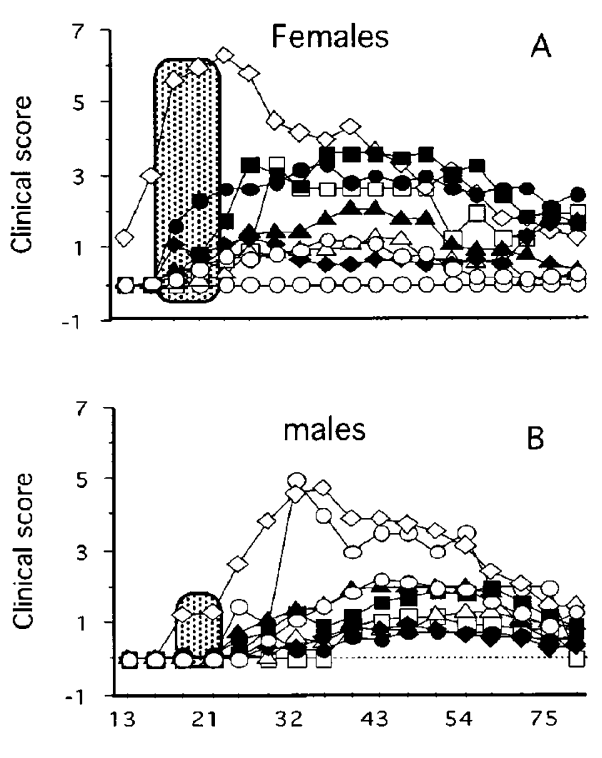

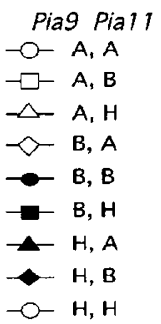

Pia9 Pial1

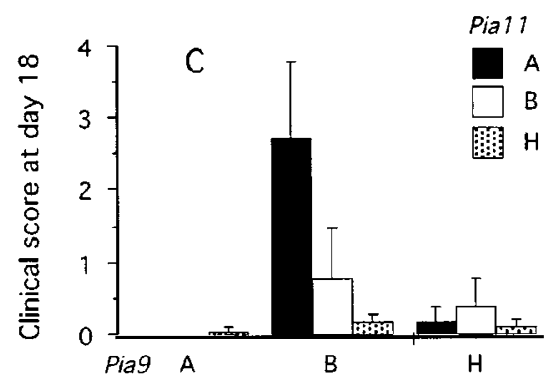

- - A, A

$\square$ A, B

$\sim A, H$

$\sim$ B, A

- $B, B$

$-\mathrm{B}, \mathrm{H}$

스 $\mathrm{H}, \mathrm{A}$

$\neg \mathrm{H}, \mathrm{B}$

$-\mathrm{O} H, \mathrm{H}$

Figure 6 Interaction between Pia9 and Pia11. (A) Clinical scores after pristane injection in the rats with different Pia9 and Pia11 genotypes in the female. Shaded area shows the significant difference $(P<0.05)$ analysed with Kruskal-Wallis test. (B) The same as $\mathbf{A}$ but in the male. (C) clinical scores at day 18 after pristane injection in all the rats with different combinations between Pia9 (Marker D1Rat51) and Pia11 (marker D16Wox8). Two-way ANOVA was used to analyse the significance and synergistic effects $(P<0.01)$.
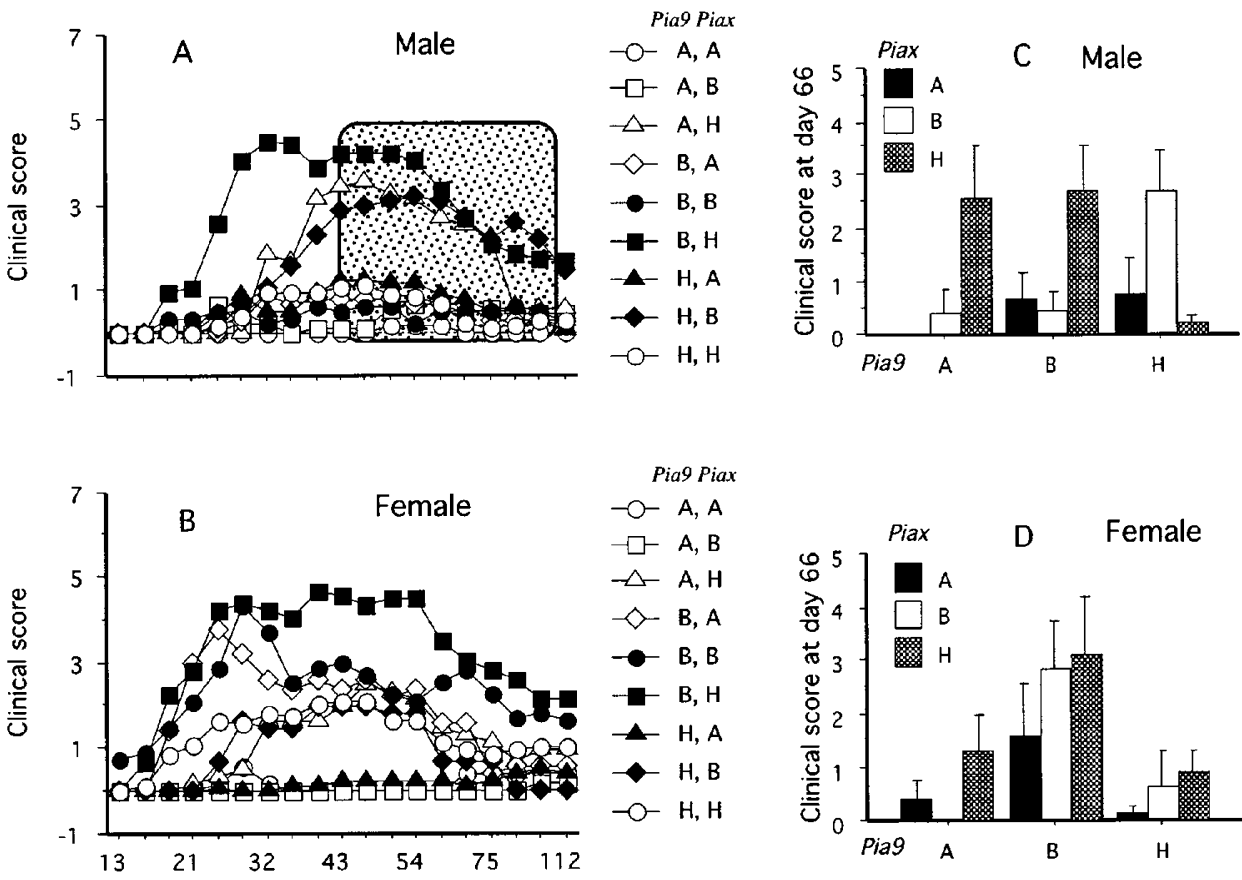

Pia9 Piax
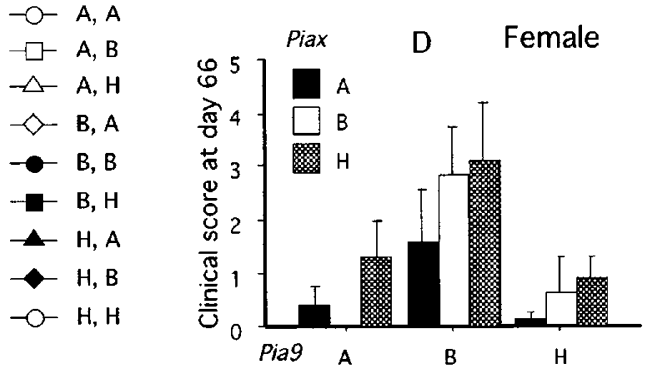

Days after pristane injection

Figure 7 Interaction between Pia9 and Piax. (A) clinical scores after pristane injection in the rats with different Pia9 and Piax genotypes in males. Shaded area shows the significant difference $(P<0.05)$ analysed with Kruskal-Wallis test. (B) The same as $\mathbf{A}$ but in females. Clinical scores at day 66 after pristane injection in males (C) and females (D) with different combinations between Pia9 (marker D1 Rat51) and Piax (marker D14Rat64). Two-way ANOVA was used to analyse the significance and synergistic effects showed in only males $(P<0.01)$ not females $(P>0.05)$. 
model. ${ }^{38}$ Another alternative is to first identify the candidate genes in the QTLs and then to study their relationship at the molecular level.

Pia9, on chromosome 1, colocalises with previously identified loci in the rat named Cia2, Eae6. ${ }^{22,26}$ In the homologous region on mouse chromosome 7 , there is a locus associated with CIA, Cia7, ${ }^{19}$ and another associated with EAE, Eae4. ${ }^{39}$ Pia4 also colocalises with other QTLs for autoimmune disease, for example, Eae5, Eau2 and Cia12. ${ }^{26,40,41}$ In a region on mouse chromosome 5, which is homologous to Pia4, association has been reported to arthritis severity of Lyme disease. ${ }^{42}$ These findings indicate that common susceptibility genes for several autoimmune diseases exist. However, there is no previously identified QTLs located in the regions of Piax and Pia11, suggesting that these may be specific for arthritis.

\section{Acknowledgements}

We thank L Lindström and Carlos Palestro for taking care of the animals and Ludmila Elfineh for expertise and assistance in genotyping. The work was supported by grants from the Beijer foundation, the King Gustaf V:s 80-year foundation, the Kock and Österlund Foundations, the Swedish Association against Rheumatism, the Swedish Medical Research Council, and EU (BMH4-97-2522).

\section{References}

1 Sangha O: Epidemiology of rheumatic diseases. Rheumatology 2000; 39 (Suppl 2): 3-12.

2 Brennan P, Silman A: Why the gender difference in susceptibility to rheumatoid arthritis?. Ann Rheum Dis 1995; 54: 694-695.

3 Zanelli E, Breedveld FC, de Vries RR: HLA class II association with rheumatoid arthritis: facts and interpretations. Hum Immunol 2000; 61: 1254-1261.

4 Claus R, Bittorf T, Walzel $\mathrm{H}$ et al: High concentration of soluble HLA-DR in the synovial fluid: generation and significance in rheumatoid-like inflammatory joint diseases. Cell Immunol 2000; 206: $85-100$.

5 Roudier J: Association of MHC and rheumatoid arthritis. Association of RA with HLA- DR4: the role of repertoire selection. Arthritis Res 2000; 2: 217-220.

6 Singal DP, Li J, Zhu Y: HLA class III region and susceptibility to rheumatoid arthritis. Clin Exp Rheumatol 2000; 18: 485-491.

7 Fife MS, Fisher SA, John S et al: Multipoint linkage analysis of a candidate gene locus in rheumatoid arthritis demonstrates significant evidence of linkage and association with the corticotropinreleasing hormone genomic region. Arthritis Rheum 2000; 43: $1673-1678$.

8 Gregersen PK, Silver J, Winchester RJ: The shared epitope hypothesis. An approach to understanding the molecular genetics of susceptibility to rheumatoid arthritis. Arthritis Rheum 1987; 30: $1205-1213$.

9 Penzotti JE, Doherty D, Lybrand TP, Nepom GT: A structural model for TCR recognition of the HLA class II shared epitope sequence implicated in susceptibility to rheumatoid arthritis. $J$ Autoimmun 1996; 9: 287-293.

10 Lahesmaa R, Skurnik M, Toivanen P: Molecular mimicry: any role in the pathogenesis of spondyloarthropathies?. Immunol Res 1993; 12: $193-208$.

11 MacGregor AJ, Snieder H, Rigby AS et al: Characterizing the quantitative genetic contribution to rheumatoid arthritis using data from twins. Arthritis Rheum 2000; 43: 30-37.
12 Brown MA, Laval SH, Brophy S, Calin A: Recurrence risk modelling of the genetic susceptibility to ankylosing spondylitis. Ann Rheum Dis 2000; 59: 883-886.

13 Wordsworth P, Bell J: Polygenic susceptibility in rheumatoid arthritis. Ann Rheum Dis 1991; 50: 343-346.

14 Vyse TJ, Todd JA: Genetic analysis of autoimmune disease. Cell 1996; 85: 311-318.

15 Jawaheer D, MacGregor AJ, Gregersen PK, Silman AJ, Ollier WE: Unexpected HLA haplotype sharing in dizygotic twin pairs discordant for rheumatoid arthritis. J Med Genet 1996; 33: $1015-1018$.

16 Cornelis F, Faure S, Martinez M et al: New susceptibility locus for rheumatoid arthritis suggested by a genome-wide linkage study. Proc Natl Acad Sci USA 1998; 95: 10746-10750.

17 Holmdahl R, Lorentzen J, Lu S et al: Arthritis induced in rats with non-immunigenic adjuvants as models for rheumatoid arthritis. Immunol Rev 2001; 184: 184-202.

18 Vingsbo-Lundberg C, Nordquist N, Olofsson P et al: Genetic control of arthritis onset, severity and chronicity in a model for rheumatoid arthritis in rats. Nat Genet 1998; 20: 401-404.

19 Yang HT, Jirholt J, Svensson L et al: Identification of genes controlling collagen-induced arthritis in mice: striking homology with susceptibility loci previously identified in the rat. J Immunol 1999; 163: 2916-2921.

20 Vingsbo C, Jonsson R, Holmdahl R: Avridine-induced arthritis in rats; a T cell-dependent chronic disease influenced both by MHC genes and by non-MHC genes. Clin Exp Immunol 1995; 99: 359 363.

21 Lorentzen JC, Glaser A, Jacobsson L et al: Identification of rat susceptibility loci for adjuvant-oil induced arthritis. Proc Natl Acad Sci USA 1998; 95: 6383-6387.

22 Remmers EF, Longman RE, Du Y et al: A genome scan localizes five non-MHC loci controlling collagen-induced arthritis in rats. Nature Genet 1996; 14: 82-85.

23 Nordquist N, Olofsson P, Vingsbo-Lundberg C, Petterson U, Holmdahl R: Complex genetic control in a rat model for rheumatoid arthritis. J Autoimmun 2000; 15: 425-432.

24 Wilder RL, Griffiths MM, Remmers EF et al: Localization in rats of genetic loci regulating susceptibility to experimental erosive arthritis and related autoimmune diseases. Transplant Proc 1999; 31: $1585-1588$.

25 Merriman TR, Cordell HJ, Eaves IA et al: Suggestive evidence for association of human chromosome 18q12-q21 and its orthologue on rat and mouse chromosome 18 with several autoimmune diseases. Diabetes 2001; 50: 184-194.

26 Bergsteinsdottir K, Yang HT, Pettersson U, Holmdahl R: Evidence for common autoimmune disease genes controlling onset, severity, and chronicity based on experimental models for multiple sclerosis and rheumatoid arthritis. J Immunol 2000; 164: $1564-$ 1568.

27 Kawahito Y, Cannon GW, Gulko PS et al: Localization of quantitative trait loci regulating adjuvant-induced arthritis in rats: evidence for genetic factors common to multiple autoimmune diseases. J Immunol 1998; 161: 4411 - 4419.

28 Jawaheer D, Seldin MF, Amos CI et al: A genomewide screen in multiplex rheumatoid arthritis families suggests genetic overlap with other autoimmune diseases. Am J Hum Genet 2001; 68 927-936.

29 Vingsbo C, Sahlstrand P, Brun JG, Jonsson R, Saxne T, Holmdahl R: Pristane-induced arthritis in rats: a new model for rheumatoid arthritis with a chronic disease course influenced by both major histocompatibility complex and non-major histocompatibility complex genes. Am J Pathol 1996; 149: 1675-1683.

30 Lander ES, Green P, Abrahamson J et al: MAPMAKER: an interactive computer package for constructing primary genetic linkage maps of experimental and natural populations. Genomics 1987; 1: $174-181$.

31 Lander E, Kruglyak L: Genetic dissection of complex traits: guidelines for interpreting and reporting linkage results. Nat Genet 1995; 11: $241-247$. 
32 Holmdahl R: Female preponderance for development of arthritis in rats is influenced by both sex chromosomes and sex steroids. Scand J Immunol 1995; 42: 104-109.

$33 \mathrm{Lu} \mathrm{S}$, Carlsen S, Hansson AS, Holmdahl R: Immunization of rats with homologous type XI collagen leads to chronic and relapsing arthritis with different genetics and joint pathology than arthritis induced with homologous type II collagen. J Autoimmun 2002, in press.

34 Taylor BA, Phillips SJ: Detection of obesity QTLs on mouse chromosomes 1 and 7 by selective DNA pooling. Genomics 1996; 34: $389-398$.

35 Carswell HV, Anderson NH, Clark JS et al: Genetic and gender influences on sensitivity to focal cerebral ischemia in the stroke-prone spontaneously hypertensive rat. Hypertension 1999; 33: $681-685$

36 Furuya T, Salstrom JL, McCall-Vining S et al: Genetic dissection of a rat model for rheumatoid arthritis significant gender influences on autosomal modifier loci. Hum Mol Genet 2000; 9: 2241-2250.

37 Griffiths MM, Cannon GW, Leonard PA, Reese VR: Induction of autoimmune arthritis in rats by immunization with homologous rat type II collagen is restricted to the RT1av1 haplotype. Arthritis Rheum 1993; 36: 254-258.
38 Morel L, Blenman KR, Croker BP, Wakeland EK: The major murine systemic lupus erythematosus susceptibility locus, Sle1, is a cluster of functionally related genes. Proc Natl Acad Sci USA 2001; 98: 1787 - 1792 .

39 Butterfield RJ, Sudweeks JD, Blankenhorn EP et al: New genetic loci that control susceptibility and symptoms of experimental allergic encephalomyelitis in inbred mice. J Immunol 1998; 161: $1860-1867$

40 Sun SH, Silver PB, Caspi RR et al: Identification of genomic regions controlling experimental autoimmune uveoretinitis in rats. Int Immunol 1999; 11: 529-534.

41 Griffiths MM, Wang J, Joe B et al: Identification of four new quantitative trait loci regulating arthritis severity and one new quantitative trait locus regulating autoantibody production in rats with collagen-induced arthritis. Arthritis Rheum 2000; 43: $1278-1289$.

42 Weis JJ, McCracken BA, Ma Y et al: Identification of quantitative trait loci governing arthritis severity and humoral responses in the murine model of Lyme disease. J Immunol 1999; 162: 948 956. 